\title{
DIFFERENTIAL EQUATIONS WITH NONLINEAR BOUNDARY CONDITIONS
}

\author{
MICHAL FEČKAN \\ (Communicated by Hal L. Smith)
}

\begin{abstract}
This paper shows the existence of multiple unbounded branches of solutions for certain equations via the Nielsen fixed-point theory.
\end{abstract}

\section{INTRODUCTION}

This paper deals with the existence of solutions of equations of the form

$$
L u=F(u),
$$

where $L: X \rightarrow Y$ is a linear Fredholm operator of positive index, $F: Y \rightarrow Y$ is continuous, $X, Y$ are Banach spaces, and $X$ is compactly embedded into $Y$. We recall that $L$ is a Fredholm operator iff $n=\operatorname{dim} \operatorname{ker} L<\infty, \operatorname{Im} L$ is closed, and $m=$ codim $\operatorname{Im} L<\infty$. The index of $L$ is defined by $n-m$.

Equations of the above form have been studied in [1-3, 9-11] for instance. Amann, Ambrosetti, and Mancini [1] used a global variant of the LjapunovSchmidt method to show the existence of infinitely many solutions of (1.1) for some specific cases. Mawhin [2] and Nirenberg [3] applied stable homotopy arguments to get existence results. Similar methods were used in [9-11].

Our approach is a continuation of [4-7], where retraction techniques of Nielsen fixed-point theory are developed to produce lower bounds for the number of solutions.

Let $X=X_{1} \oplus \operatorname{ker} L$ and $X_{1}$ be a closed linear subspace. The main assumption on $F$ is the following:

$$
\begin{aligned}
& F \text { is bounded, i.e., }|F(u)|<c, \forall u \in X \text {, and there exists } \\
& \lim _{r \rightarrow+\infty} P F\left(u_{1}+\text { r. } u_{2}\right)=\phi\left(u_{2}\right) \text { uniformly for } u_{2} \in S^{n-1}=
\end{aligned}
$$$$
\{u \in \operatorname{ker} L|| u \mid=1\} \text { and } u_{1} \in X_{1} \text { bounded, }
$$

where $P: Y \rightarrow Y_{1}$ is a continuous projection and $Y=Y_{1} \oplus \operatorname{Im} L$.

We shall show that if $\phi$ has suitable properties on a compact locally contractible subset $K \subset S^{n-1}, K \neq S^{n-1}$, then there is a natural number $p$ such that for each $r$ sufficiently large the equation (1.1) possesses at least $p$ solutions of the form $u=u_{1}+u_{2}, u_{1} \in X_{1}, u_{2} \in \operatorname{ker} L,\left|u_{2}\right|=r$. Hence (1.1) has at least $p$ unbounded branches of solutions.

Received by the editors August 3, 1992.

1991 Mathematics Subject Classification. Primary 34B15. 
In $\S 2$, we consider the abstract setting of (1.1). It will also be shown that the assumption (1.2) can be weakened. Several examples are given in $\S 3$.

\section{THE ABSTRACT FRAMEWORK}

First we need the following

Definition 2.1 [7]. Suppose that $\Pi: W \rightarrow A$ is a map, $W, A$ are subsets of $Z$, and $A \subset W$. If $\Pi(a)=a$ for each $a \in A$, then $\Pi$ is called a retraction of $W$ to $A$ and $A$ is called a retract of $W$.

Definition 2.2 ([7]). Let $Z$ be a normed linear space with norm denoted by $|$.$| .$ Suppose that $T: Z \rightarrow Z$ is a map and $W$ is a subset that retracts onto a subset $Q$ of itself by a retraction $\Pi: W \rightarrow Q$. We shall say that $T$ is $\mu$-retractible onto $Q$ with a retraction $\Pi$ if it holds

$$
\{x \in Z \mid \text { there exists } a \in T(Q) \text { such that }|x-a|<\mu\} \subset W
$$

and

$$
\text { if } y \in W \backslash Q, \Pi(y)=x \text {, then }|y-T(x)|>\mu \text {. }
$$

We shall say that $T$ is retractible onto $Q$ with a retraction $\Pi$ if $T(Q) \subset W$, and if $y \in W \backslash Q, \Pi(y)=x$, then $y \neq T(x)$.

We see that if $T$ is $\mu$-retractible onto $Q$, then any $\mu$-perturbation of $T$ is still retractible onto $Q$. The main advantage of Definition 2.2 is the following: If $T$ is retractible onto $Q$ with a retraction $\Pi: W \rightarrow Q$, then the map $\Pi \cdot T: Q \rightarrow Q$ has a fixed point $x \in Q$ if and only if $T(x)=x$.

From now on, all maps are supposed to be continuous.

Since $L$ is Fredholm, we have $Y=Y_{1} \oplus \operatorname{Im} L, \operatorname{dim} Y_{1}=m$. Let $P: Y \rightarrow$ $Y_{1}$ be a continuous projection; then (1.1) has the form

$$
\begin{array}{ll}
L u_{1}=(I-P) F\left(u_{1}+r . u_{2}\right), & u_{1} \in X_{1}, u_{2} \in S^{n-1} ; \\
0=P F\left(u_{1}+r . u_{2}\right), \quad r>0 . &
\end{array}
$$

Since $L: X_{1} \rightarrow \operatorname{Im} L$ is invertible, we have

$$
\begin{gathered}
u_{1}=L^{-1}(I-P) F\left(u_{1}+r . u_{2}\right), \\
0=P F\left(u_{1}+r . u_{2}\right) .
\end{gathered}
$$

We know that $X$ is compactly embedded into $Y$. Thus the operator $\left(u_{1}, u_{2}\right) \rightarrow$ $L^{-1}(I-P) F\left(u_{1}+r . u_{2}\right)$ is compact and bounded as well, since $F$ is bounded by (1.2). Let $K \subset S^{n-1}, K \neq S^{n-1}$, be a compact locally contractible subset.

Since $K \neq S^{n-1}$, we do consider that $K$ is embedded into $\mathbb{R}^{n-1}$, i.e., $K$ is a subset of $\mathbb{R}^{n-1}$.

Theorem 2.3. Assume that the above assumptions are satisfied, and let $S$ : $\operatorname{Im} P \rightarrow \mathbb{R}^{m}$ be an isomorphism. We denote by $\tilde{\phi}$ the composition of the map $S \phi: K \rightarrow \mathbb{R}^{m}$, where $\phi$ is defined in (1.2), with an inclusion $\eta$ of $\mathbb{R}^{m}$ into $\mathbb{R}^{n-1}$ (note $m<n$ ). Let Id: $\mathbb{R}^{n-1} \rightarrow \mathbb{R}^{n-1}$ be the identity.

If $\mathrm{Id}+\tilde{\phi}$ is $\mu$-retractible onto $K$ with a retraction $\Pi$, then there is a number $r_{0}>0$ such that for any $r \geq r_{0}$ the equation (1.1) possesses at least $N(\Pi \cdot(\mathrm{Id}+\tilde{\phi}))($ the Nielsen number of the map $\Pi \cdot(\mathrm{Id}+\tilde{\phi}): K \rightarrow K)$ solutions of the form $u=u_{1}+u_{2}, u_{1} \in X_{1}, u_{2} \in \operatorname{ker} L,\left|u_{2}\right|=r$. 
Proof. We shall solve (2.2a), (2.2b). Let $\rho_{M}: X_{1} \rightarrow B_{M}=\left\{x_{1} \in X_{1}|| x \mid \leq M\right\}$ be the usual retraction [7] defined as follows:

$$
\rho_{M}(x)= \begin{cases}x, & x \in B_{M} ; \\ \frac{x}{|x|} \cdot M, & x \notin B_{M} .\end{cases}
$$

Since $u_{2} \in K \subset S^{n-1}, K \neq S^{n-1}$, we consider $u_{2}$ as an element of $\mathbb{R}^{n-1}$. Then we modify $(2.2 \mathrm{a}),(2.2 \mathrm{~b})$ in the form

$$
\begin{aligned}
& u_{1}=\rho_{M}\left(L^{-1}(I-P) F\left(u_{1}+r . u_{2}\right)\right), \\
& u_{2}=\Pi\left(u_{2}+\eta S P F\left(u_{1}+r . u_{2}\right)\right)
\end{aligned}
$$

for $M, r$ sufficiently large.

Consider the map

$$
\begin{gathered}
H_{r, t}\left(u_{1}, u_{2}\right)=\left(\rho_{M}\left(t \cdot L^{-1}(I-P) F\left(u_{1}+r . u_{2}\right)\right), \Pi\left(u_{2}+\eta S P F\left(u_{1}+r . u_{2}\right)\right)\right), \\
H_{r, t}: B_{M} \times K \rightarrow B_{M} \times K, \quad t \in[0,1] .
\end{gathered}
$$

Of course, the fixed points of $H_{r, 1}$ are solutions of (2.3). We claim that for $M, r$ sufficiently large the fixed points of $H_{r, 1}$ are solutions of (2.2a), (2.2b). Let us assume (2.3) holds. Then for a fixed number $M>0$ large enough we have

$$
\left|L^{-1}(I-P) F\left(u_{1}+r . u_{2}\right)\right|<M .
$$

Hence $u_{1}=L^{-1}(I-P) F\left(u_{1}+r \cdot u_{2}\right)$. Moreover, we have

$$
\lim _{r \rightarrow+\infty} \eta S P F\left(u_{1}+r . u_{2}\right)=\eta S \phi\left(u_{2}\right)
$$

uniformly for $u_{2} \in K, u_{1} \in B_{M}$. We know that $u_{2}+\eta S \phi\left(u_{2}\right)=u_{2}+\tilde{\phi}\left(u_{2}\right)$ is $\mu$-retractible onto $K$ with the retraction $\Pi$. But then for any $r$ large the map $u_{2} \rightarrow u_{2}+\eta S P F\left(u_{1}+r . u_{2}\right)$ is retractible onto $K$ with the retraction $\Pi$ as well. Hence

$$
u_{2}=u_{2}+\eta S P F\left(u_{1}+r \cdot u_{2}\right)
$$

and we have proved the assertion.

On the other hand, $N\left(H_{r, 1}\right)=N\left(H_{\infty}, 0\right)$, where

$$
\begin{gathered}
H_{\infty, 0}: B_{M} \times K \rightarrow B_{M} \times K, \\
H_{\infty, 0}\left(u_{1}, u_{2}\right)=\left(0, \Pi\left(u_{2}+\tilde{\phi}\left(u_{2}\right)\right)\right) .
\end{gathered}
$$

We note that $B_{M} \times K$ is an ANR [7] and $H_{r, t}$ are compact operators. It is clear [7] that $N\left(H_{\infty, 0}\right)=N(\Pi \cdot(\mathrm{Id}+\tilde{\phi}))$. Summarizing we see that for any $r$ sufficiently large and for a fixed number $M$ large enough, the map $H_{r, 1}$ has at least $N(\Pi \cdot(\mathrm{Id}+\tilde{\phi}))$ fixed points in $B_{M} \times K$. But we have shown that these fixed points are solutions of (1.1) as well. This completes the proof.

We see that the choice of $\tilde{\phi}$ is not unique. It depends on the embedding both of $K$ into $\mathbb{R}^{n-1}$ and $\mathbb{R}^{m}$ into $\mathbb{R}^{n-1}$. We shall always refer to a specific choice.

Corollary 2.4. Suppose the validity of assumptions of Theorem 2.3. Moreover, let $(\operatorname{Id}+\lambda . \tilde{\phi})(K) \subset \operatorname{dom} \Pi$, for any $\lambda \in[0,1]$. Here dom $\Pi$ is the domain of definition of $\Pi$. 
If $\chi(K) \neq 0$, the Euler-Poincare characteristic of $K$, then for any $r>0$ large the equation (1.1) has a solution of the form $u=u_{1}+u_{2}, u_{1} \in X_{1}, u_{2} \in$ $\operatorname{ker} L,\left|u_{2}\right|=r$.

Proof. We shall compute $N(\Pi \cdot(\mathrm{Id}+\tilde{\phi}))$. Since $\mathrm{Id}+\lambda . \tilde{\phi}: K \rightarrow$ dom $\Pi$ holds for any $\lambda \in[0,1]$, we have

$$
N(\Pi \cdot(\mathrm{Id}+\tilde{\phi}))=N(\mathrm{Id}) .
$$

Using $\chi(K) \neq 0$ (see [8]) we obtain $N(\mathrm{Id}) \neq 0$. The proof is finished.

Of course, finding the set $K$ with the retraction $\Pi$ and then computing the Nielsen number $N(\Pi \cdot(\operatorname{Id}+\tilde{\phi}))$ is generally a difficult task. In the next section, we shall follow the reverse approach. We shall construct differential equations satisfying the assumptions of Theorem 2.3.

We see from the proof of Theorem 2.3 that condition (1.2) can be weakened to the following:

(c1) $(I-P) F$ is bounded, i.e., $|(I-P) F(u)| \leq c, \forall u \in X$.

(c2) There exists a continuous map $\phi: S^{n-1} \rightarrow \mathbb{R}^{m}$ satisfying

(a) $\tilde{\phi}$ is $\mu$-retractible onto $K$ with a retraction $\Pi$, where $K$ has the above properties and $\tilde{\phi}$ is the composition of the map $\phi: K \rightarrow \mathbb{R}^{m}$ with an inclusion $\eta$ of $\mathbb{R}^{m}$ to $\mathbb{R}^{n-1}$, and

(b) $\lim \sup _{r \rightarrow+\infty}\left|u_{2}+\eta \operatorname{SPF}\left(u_{1}+r \cdot u_{2}\right)-\tilde{\phi}\left(u_{2}\right)\right|<\mu$ uniformly for $u_{2} \in$ $K \subset \mathbb{R}^{n-1}$ and $u_{1}$ from bounded subsets.

Hence the condition (c2)(b) allows the map Id $+\eta S P F$ asymptotically to oscillate near $\tilde{\phi}$ on the set $K \subset \mathbb{R}^{n-1}$ with a deviation less then $\mu$. Moreover, the higher the $\mu$-retractibility of $\tilde{\phi}$, the higher the possible deviation of the asymptotic oscillation of $\operatorname{Id}+\eta S P F$ near $\tilde{\phi}$. This means that a measure of the set of all maps $F$ satisfying the above conditions increases with the number $\mu$.

Now we give the following two special cases of (1.1):

$$
\begin{array}{ll}
L_{1} x=N_{1}(x), & B_{1}(x)=0 ; \\
L_{2} x=N_{2}(x, \lambda), & B_{2}(x)=0,
\end{array}
$$

where $L_{1}, L_{2}: \tilde{X} \rightarrow \tilde{Y}$ are linear Fredholm operators such that $L_{2}$ is invertible and codim $\operatorname{Im} L_{1}=0, \operatorname{dim} \operatorname{ker} L_{1}=q$. Furthermore, $N_{1}: \tilde{X} \rightarrow \tilde{Y}, N_{2}: \tilde{X} \times$ $\mathbb{R}^{k} \rightarrow \tilde{Y}$ are continuous compact bounded and $B_{1}: \tilde{X} \rightarrow \mathbb{R}^{k}, B_{2}: \tilde{X} \rightarrow \mathbb{R}^{p}$ $(0<p<k<q)$ are continuous bounded. $\tilde{X}, \tilde{Y}$ are Banach spaces and $\tilde{X}$ is compactly embedded into $\tilde{Y}$. Note that (2.a) and (2.b) are studied in [2] and [7], respectively.

Setting for the case (2.a)

$$
\begin{gathered}
X=\tilde{X}, \quad Y=\tilde{Y} \times \mathbb{R}^{k}, \\
L=\left(L_{1}, 0\right), \quad F=\left(N_{1}, B_{1}\right),
\end{gathered}
$$

and for the case (2.b)

$$
\begin{gathered}
X=\tilde{X} \times \mathbb{R}^{k}, \quad Y=\tilde{Y} \times \mathbb{R}^{p}, \\
L=\left(L_{2}, 0\right), \quad F=\left(N_{2}, B_{2} \cdot L_{2}^{-1} \cdot N_{2}\right),
\end{gathered}
$$

respectively, we see that (2.a), (2.b) have a form like (1.1). Applying Theorem 2.3 we obtain 
Theorem 2.5. Assume $\lim _{r \rightarrow+\infty} B_{1}\left(x_{1}+r . x_{2}\right)=\phi\left(x_{2}\right) \in \mathbb{R}^{k}$ uniformly for $x_{2} \in$ $S^{q-1}=\left\{u \in \operatorname{ker} L_{1}|| u \mid=1\right\}$ and for $x_{1} \in X_{1}$ bounded, where $\tilde{X}=X_{1} \oplus \operatorname{ker} L_{1}$. Let $K \subset S^{q-1}, K \neq S^{q-1}$ be a compact locally contractible subset. For this reason, we consider that $K$ is embedded into $\mathbb{R}^{q-1}$, i.e., $K \subset \mathbb{R}^{q-1}$. We denote by $\tilde{\phi}$ the composition of the map $\phi: K \rightarrow \mathbb{R}^{k}$, defined over, with an inclusion of $\mathbb{R}^{k}$ into $\mathbb{R}^{q-1}($ note $q>k)$. Let Id: $\mathbb{R}^{q-1} \rightarrow \mathbb{R}^{q-1}$ be the identity.

If $\mathrm{Id}+\tilde{\phi}$ is $\mu$-retractible onto $K$ with a retraction $\Pi$, then (2.a) has at least $N(\Pi \cdot(\mathrm{Id}+\tilde{\phi}))$ solutions of the form $x=x_{1}+x_{2}, x_{1} \in X_{1}, x_{2} \in \operatorname{ker} L_{1},\left|x_{2}\right|=r$ for any $r>0$ sufficiently large.

Theorem 2.6. Assume $\lim _{r \rightarrow+\infty} B_{2} L_{2}^{-1} N_{2}\left(x, r . \lambda_{2}\right)=\phi\left(\lambda_{2}\right) \in \mathbb{R}^{p}$ uniformly for $\lambda_{2} \in S^{k-1}=\left\{\lambda \in \mathbb{R}^{k}|| \lambda \mid=1\right\}$ and $x \in \tilde{X}$ bounded. Let $K \subset S^{k-1}, K \neq S^{k-1}$ be a compact locally contractible subset. For this reason, we consider that $K$ is embedded into $\mathbb{R}^{k-1}$, i.e., $K \subset \mathbb{R}^{k-1}$. We denote by $\tilde{\phi}$ the composition of the map $\phi: K \rightarrow \mathbb{R}^{p}$, defined over, with an inclusion of $\mathbb{R}^{p}$ into $\mathbb{R}^{k-1}$ (note $k>p$ ). Let Id: $\mathbb{R}^{k-1} \rightarrow \mathbb{R}^{k-1}$ be the identity.

If $\mathrm{Id}+\tilde{\phi}$ is $\mu$-retractible onto $K$ with a retraction $\Pi$, then for each $r>0$ sufficiently large the equation (2.b) has a solution at least for $N(\Pi \cdot(\mathrm{Id}+\tilde{\phi}))$ parameters $\lambda$ satisfying $|\lambda|=r$.

Similarly, we can study the equation

$$
L_{3} x=N_{3}(x, y), \quad L_{4} y=N_{4}(x, y),
$$

where $L_{3}: \tilde{X}_{1} \rightarrow \tilde{Y}_{1}, L_{4}: \tilde{X}_{2} \rightarrow \tilde{Y}_{2}$ are Fredholm operators such that the index of $L_{3}$ is negative, codim $\operatorname{Im} L_{4}=0, \operatorname{dim} \operatorname{ker} L_{4} \geq \operatorname{codim} \operatorname{Im} L_{3} . \tilde{X}_{i}$ are compactly embedded into $\tilde{Y}_{i}, i=1,2$. The appropriate limit condition is the following:

$$
\lim _{r \rightarrow+\infty} P_{3} N_{3}\left(x_{1}+r . x_{2}, y_{1}+r . y_{2}\right)=\phi\left(x_{2}, y_{2}\right),
$$

uniformly for $x_{2} \in \operatorname{ker} L_{3}, y_{2} \in \operatorname{ker} L_{4},\left|x_{2}\right|+\left|y_{2}\right|=1$, and $x_{1} \in \tilde{X}_{3}, y_{1} \in \tilde{X}_{4}$ bounded. Here $P_{3}: \tilde{Y}_{1} \rightarrow \tilde{Y}_{3}$ is a continuous projection and

$$
\tilde{X}_{1}=\tilde{X}_{3} \oplus \operatorname{ker} L_{3}, \quad \tilde{X}_{2}=\tilde{X}_{4} \oplus \operatorname{ker} L_{4}, \quad \tilde{Y}_{1}=\tilde{Y}_{3} \oplus \operatorname{Im} L_{3} .
$$

Remark 2.7. Of course, we can replace the above limit conditions by conditions similar to $(c 2)(b)$.

\section{EXAMPLES}

In this section we give several illustrative examples. Let us consider the equation

$$
\begin{aligned}
& x_{1}^{\prime}=f_{1}\left(x_{1}, x_{2}, x_{3}, t\right), \\
& x_{2}^{\prime}=f_{2}\left(x_{1}, x_{2}, x_{3}, t\right), \\
& x_{3}^{\prime}=f_{3}\left(x_{1}, x_{2}, x_{3}, t\right), \quad t \in[0,1], \\
& \mathscr{B},
\end{aligned}
$$

where $f_{1}, f_{2}, f_{3}: \mathbb{R}^{3} \times[0,1] \rightarrow \mathbb{R}$ are continuous bounded and $\mathscr{B}$ represents value conditions. We shall vary $\mathscr{B}$ to obtain the problems $(2 . a),(2 . b),(2 . c)$, respectively. 
First of all, we shall construct a mapping $\tau: \mathscr{A}_{1,3} \rightarrow \mathbb{R}^{2}$ such that $\tau$ is $\mu$ retractible onto $\mathscr{A}_{1,3}$ with the retraction $\rho_{1,3}$. Here

$$
\begin{gathered}
\mathscr{A}_{1,3}=\left\{(\rho, \omega) \in \mathbb{R}_{+} \times S^{1}|1 \leq| \rho \mid \leq 3\right\} ; \quad \rho_{1,3}: \mathbb{R}_{+} \times S^{1} \rightarrow \mathscr{A}_{1,3} ; \\
\rho_{1,3}(\rho, \omega)= \begin{cases}(\rho, \omega), & 1 \leq \rho \leq 3, \\
(1, \omega), & 0<\rho<1, \\
(3, \omega), & 3<\rho .\end{cases}
\end{gathered}
$$

We note that $(\rho, \omega)$ are the polar coordinates on $\mathbb{R}^{2}$ and $\mathscr{A}_{1,3}$ is the annulus. Let $\alpha: \mathbb{R}_{+} \rightarrow \mathbb{R}_{+}, \beta: S^{1} \rightarrow S^{1}$ be continuous maps such that

$$
\alpha(1)>1+\mu, \quad \alpha(3)<3-\mu, \quad \alpha(\rho)>\mu \quad \forall \rho \in[1,3] .
$$

In the polar coordinates, consider the map

$$
\tau(\rho, \omega)=(\alpha(\rho), \beta(\omega)) .
$$

Then $|\tau(\rho, \omega)|=\alpha(\rho)$ and by [7], $\tau: \mathscr{A}_{1,3} \rightarrow \mathbb{R}^{2}$ is $\mu$-retractible onto $\mathscr{A}_{1,3}$ with the retraction $\rho_{1,3}$. We also know from [7] that $N\left(\rho_{1,3} \cdot \tau\right)=|\operatorname{deg} \beta-1|$, where $\operatorname{deg} \beta$ is the homotopy degree of the map $\beta: S^{1} \rightarrow S^{1}$.

Since $S^{2}$ is the compactification of $\mathbb{R}^{2}$ and $\mathscr{A}_{1,3}$ is a bounded closed subset of $\mathbb{R}^{2}$, we continuously extend the map $(\tau-\mathrm{Id}): U \rightarrow \mathbb{R}^{2}$ on $S^{2}$. Here Id: $\mathbb{R}^{2} \rightarrow \mathbb{R}^{2}$ is the identity and $U \subset \mathbb{R}^{2} \backslash\{(\rho, \omega) \mid \rho \geq 1 / 2\}$ is a bounded open neighbourhood of $\mathscr{A}_{1,3}$. Let $\phi$ be an extension. Hence $\phi / U=\tau-$ Id.

Finally, we put

$$
\begin{aligned}
\mathscr{M}_{\phi}=\left\{B \in C^{0}\left(\mathbb{R}_{+} \times S^{2}, \mathbb{R}^{2}\right) \mid B\right. \text { is bounded and } \\
\limsup _{r \rightarrow+\infty}|B(r, y)-\phi(y)|<\mu \text { uniformly for } y \in U \subset S^{2}, \\
\left.U \text { is the above bounded neighbourhood of } \mathscr{A}_{1,3}\right\} .
\end{aligned}
$$

Furthermore, we identify the set $\mathbb{R}_{+} \times S^{2}$ with $\left\{z \in \mathbb{R}^{3} \mid z \neq 0\right\}$ in the following way $r=|z| \in \mathbb{R}_{+}, y=z /|z| \in S^{2}$. Hence we consider

$$
C^{0}\left(\mathbb{R}_{+} \times S^{2}, \mathbb{R}^{2}\right) \supset C^{0}\left(\mathbb{R}^{3}, \mathbb{R}^{2}\right) .
$$

Now we return to (3.1). We note that we shall always identify a constant function $x()=$.$a with the number a$.

A. Let

$$
\mathscr{B}=\left\{B\left(x_{1}(0), x_{2}(1), x_{3}(1)\right)=0\right\},
$$

where $B \in \mathscr{M}_{\phi} \cap C^{0}\left(\mathbb{R}^{3}, \mathbb{R}^{2}\right.$ ) (see (3.2)). We put

$$
\begin{aligned}
& \tilde{X}=C^{1}\left([0,1], \mathbb{R}^{3}\right), \tilde{Y}=C^{0}\left([0,1], \mathbb{R}^{3}\right), \\
& L_{1}\left(x_{1}, x_{2}, x_{3}\right)=\left(x_{1}^{\prime}, x_{2}^{\prime}, x_{3}^{\prime}\right), \\
& N_{1}\left(x_{1}, x_{2}, x_{3}\right)=\left(f_{1}\left(x_{1}, x_{2}, x_{3}, .\right), f_{2}\left(x_{1}, x_{2}, x_{3}, .\right), f_{3}\left(x_{1}, x_{2}, x_{3}, .\right)\right), \\
& B_{1}\left(x_{1}, x_{2}, x_{3}\right)=B\left(x_{1}(0), x_{2}(1), x_{3}(1)\right) .
\end{aligned}
$$

Then (3.1) has the form (2.a). Applying Theorem 2.5 and Remark 2.7 we obtain 
Theorem 3.1. In case $\mathrm{A}$, equation (3.1) has at least $|\operatorname{deg} \beta-1|$ unbounded branches of solutions.

Proof. In this case, $\operatorname{dim} \operatorname{ker} L_{1}=q=3$ and $k=2$ in Theorem 2.5. Hence $\mathbb{R}^{k}=\mathbb{R}^{q-1}$. Moreover, we know that

$$
\limsup _{r \rightarrow+\infty}\left|B\left(r . c_{1}, r . c_{2}, r . c_{3}\right)-\phi\left(c_{1}, c_{2}, c_{3}\right)\right|<\mu
$$

uniformly for $\left(c_{1}, c_{2}, c_{3}\right) \in U$, where $U \subset S^{2}$ is the above bounded neighbourhood of $\mathscr{A}_{1,3}$. But this relation also implies

$$
\limsup _{r \rightarrow+\infty}\left|B\left(a_{1}+r . c_{1}, a_{2}+r . c_{2}, a_{3}+r . c_{3}\right)-\phi\left(c_{1}, c_{2}, c_{3}\right)\right|<\mu
$$

uniformly for $\left(a_{1}, a_{2}, a_{3}\right)$ from a bounded subset and $\left(c_{1}, c_{2}, c_{3}\right) \in \mathscr{A}_{1,3}$. Using Theorem 2.5 and Remark 2.7, the proof is finished.

B. Let

$$
\mathscr{B}=\left\{x_{1}(0)=x_{1}(1), g\left(x_{1}(0), x_{2}(0), x_{3}(1)\right)=0\right\},
$$

where $g: \mathbb{R}^{3} \rightarrow \mathbb{R}$ is continuous bounded. We put

$$
\begin{aligned}
& \tilde{X}_{1}=\left\{x_{1} \in C^{1}([0,1], \mathbb{R}) \mid x_{1}(0)=x_{1}(1)\right\}, \quad \tilde{Y}_{1}=C^{0}([0,1], \mathbb{R}) \times \mathbb{R}, \\
& \tilde{X}_{2}=C^{1}\left([0,1], \mathbb{R}^{2}\right), \quad \tilde{Y}_{2}=C^{0}\left([0,1], \mathbb{R}^{2}\right), \\
& L_{3} x_{1}=\left(x_{1}^{\prime}, 0\right), \\
& N_{3}\left(x_{1}, x_{2}, x_{3}\right)=\left(f_{1}\left(x_{1}, x_{2}, x_{3}, .\right), g\left(x_{1}(0), x_{2}(0), x_{3}(1)\right)\right), \\
& L_{4}\left(x_{2}, x_{3}\right)=\left(x_{2}^{\prime}, x_{3}^{\prime}\right), \\
& N_{4}\left(x_{1}, x_{2}, x_{3}\right)=\left(f_{2}\left(x_{1}, x_{2}, x_{3}, .\right), f_{3}\left(x_{1}, x_{2}, x_{3}, .\right)\right), \\
& P_{3}\left(x_{1}, c\right)=\left(\int_{0}^{1} x_{1}(s) d s, c\right) .
\end{aligned}
$$

Then (3.1) has the form (2.c).

Theorem 3.2. Assume the map

$$
\left(c_{1}, c_{2}, c_{3}\right) \rightarrow\left(f_{1}\left(c_{1}, c_{2}, c_{3}, s\right), g\left(c_{1}, c_{2}, c_{3}\right)\right) \equiv B\left(c_{1}, c_{2}, c_{3}, s\right)
$$

belongs to $\mathscr{M}_{\phi} \cap C^{0}\left(\mathbb{R}^{3}, \mathbb{R}^{2}\right)$ for all $s \in[0,1]$ and satisfies the condition

$$
\limsup _{r \rightarrow+\infty}|B(r, y, s)-\phi(y)|<\mu
$$

uniformly for $y \in U \subset S^{2}$ and $s \in[0,1]$ (see (3.2)).

Then (3.1), for the case $\mathrm{B}$, has at least $|\operatorname{deg} \beta-1|$ unbounded branches of solutions.

Proof. We know that

$$
\underset{r \rightarrow+\infty}{\limsup }\left|\left(f_{1}\left(r . c_{1}, r . c_{2}, r . c_{3}, s\right), g\left(r . c_{1}, r . c_{2}, r . c_{3}\right)\right)-\phi\left(c_{1}, c_{2}, c_{3}\right)\right|<\mu
$$


uniformly for $\left(c_{1}, c_{2}, c_{3}\right) \in U, s \in[0,1]$, where $U \subset S^{2}$ is the above bounded neighbourhood of $\mathscr{A}_{1,3}$. Hence

$$
\begin{aligned}
\limsup _{r \rightarrow+\infty} \mid & \mid\left(\int_{0}^{1} f_{1}\left(a_{1}(s)+r . c_{1}, a_{2}(s)+r . c_{2}, a_{3}(s)+r . c_{3}, s\right) d s,\right. \\
& \left.\quad g\left(a_{1}(0)+r . c_{1}, a_{2}(0)+r . c_{2}, a_{3}(1)+r . c_{3}\right)\right)-\phi\left(c_{1}, c_{2}, c_{3}\right) \mid<\mu
\end{aligned}
$$

uniformly for $\left(a_{1}, a_{2}, a_{3}\right)$ from a bounded subset and $\left(c_{1}, c_{2}, c_{3}\right) \in \mathscr{A}_{1,3}$. Using (2.4) and Remark 2.7, the proof is finished.

C. In this case we assume that $f_{i}=f_{i}\left(x_{1}, x_{2}, x_{3}, t, \lambda\right), i=1,2,3$, depend continuously also on a parameter $\lambda \in \mathbb{R}^{3}$ and they are bounded functions. Now we take

$$
\mathscr{B}=\left\{x_{1}(0)=0, x_{2}(0)=0, x_{3}(0)=0, x_{1}(1 / 2)=x_{2}(1 / 2)=0\right\}
$$

Putting

$$
\begin{aligned}
& \tilde{X}=\left\{\left(x_{1}, x_{2}, x_{3}\right) \in C^{1}\left([0,1], \mathbb{R}^{3}\right) \mid x_{1}(0)=x_{2}(0)=x_{3}(0)=0\right\}, \\
& \tilde{Y}=C^{0}\left([0,1], \mathbb{R}^{3}\right), \\
& L_{2}\left(x_{1}, x_{2}, x_{3}\right)=\left(x_{1}^{\prime}, x_{2}^{\prime}, x_{3}^{\prime}\right), \\
& N_{2}\left(x_{1}, x_{2}, x_{3}, \lambda\right)=\left(f_{1}\left(x_{1}, x_{2}, x_{3}, ., \lambda\right), f_{2}\left(x_{1}, x_{2}, x_{3}, ., \lambda\right),\right. \\
& \left.B_{2}\left(x_{1}, x_{1}, x_{2}, x_{3}, ., \lambda\right)\right), \\
& \left.\quad x_{1}(1 / 2), x_{2}(1 / 2)\right),
\end{aligned}
$$

we see that equation (3.1), for this case $C$, has the form (2.b). We note

$$
L_{2}^{-1}\left(h_{1}, h_{2}, h_{3}\right)=\left(\int_{0}^{x} h_{1}(s) d s, \int_{0}^{x} h_{2}(s) d s, \int_{0}^{x} h_{3}(s) d s\right) \text {. }
$$

We shall use Theorem 2.6 and Remark 2.7 to obtain

Theorem 3.3. Assume

$$
\limsup _{r \rightarrow+\infty}\left|f_{i}\left(c_{1}, c_{2}, c_{3}, t, r . \lambda_{1}\right)-2 . \phi_{i}\left(\lambda_{1}\right)\right|<\mu, \quad i=1,2, \phi=\left(\phi_{1}, \phi_{2}\right),
$$

uniformly for $\left(c_{1}, c_{2}, c_{3}\right)$ from bounded subsets of $\mathbb{R}^{3}, t \in[0,1]$, and $\lambda_{1} \in U$, where $U \subset S^{2}$ is the above bounded neighbourhood of $\mathscr{A}_{1,3}$.

Then for any $r>0$ large equation (3.1), in the case $\mathrm{C}$, has a solution at least for $|\operatorname{deg} \beta-1|$ parameters $\lambda$ such that $|\lambda|=r$.

Proof. In this case, $k=3, p=2$ in Theorem 2.6. Hence $\mathbb{R}^{k-1}=\mathbb{R}^{p}$. Moreover, we have

$$
\begin{aligned}
B_{2} L_{2}^{-1} & N_{2}\left(x_{1}, x_{2}, x_{3}, \lambda\right) \\
& =\left(\int_{0}^{1 / 2} f_{1}\left(x_{1}, x_{2}, x_{3}, s, \lambda\right) d s, \int_{0}^{1 / 2} f_{2}\left(x_{1}, x_{2}, x_{3}, s, \lambda\right) d s\right) .
\end{aligned}
$$


Using the assumption of Theorem 3.3, we obtain similarly as in the above proofs

$$
\underset{r \rightarrow+\infty}{\limsup }\left|B_{2} L_{2}^{-1} N_{2}\left(x, r . \lambda_{2}\right)-\phi\left(\lambda_{2}\right)\right|<\mu
$$

uniformly for $x \in \tilde{X}$ bounded, $\lambda_{2} \in \mathscr{A}_{1,3}$. Applying Theorem 2.6 and Remark 2.7 , the proof is finished.

\section{ACKNOWLEDGMENT}

The author thanks the referee for a careful reading of the manuscript.

\section{REFERENCES}

1. H. Amann, A. Ambrosetti, and G. Mancini, Elliptic equations with noninvertible Fredholm linear part and bounded nonlinearities, Math. Z. 158 (1978), 179-194.

2. J. Mawhin, Stable homotopy and ordinary differential equations with nonlinear boundary conditions, Rocky Mountain J. Math. 7 (1977), 417-424.

3. L. Nirenberg, Topics in nonlinear functional analysis, New York Univ. Lecture Notes, 1973-1974.

4. M. Fečkan, Nielsen fixed point theory and nonlinear equations, J. Differential Equations (to appear).

5. __ Multiple periodic solutions of small vector fields on differentiable manifolds, J. Differential Equations (to appear).

6. $\ldots$, Multiple perturbed solutions near nondegenerate manifolds, Comment. Math. Univ. Carolin. (to appear).

7. R. F. Brown, Topological identification of multiple solutions to parametrized nonlinear equations, Pacific J. Math. 131 (1988), 51-69.

8. _ The Lefschetz Fixed Point Theorem, Scott, Foresmann \& Co., Chicago, IL, 1971.

9. J. Tavantiz, Topological methods for finding nontrivial solutions of elliptic boundary value problems, Nonlinear Anal. T.M.A. 1 (1977), 633-649.

10. J. Mawhin and K. P. Rybakowski, Continuation theorems for semilinear equations in Banach spaces: A survey, Nonlinear Analysis (Th. M. Rassias, ed.), World Scientific, Singapore, 1987, pp. 367-405.

11. E. N. Dancer, On the existence of zeros of perturbed operators, Nonlinear Anal. T.M.A. 7 (1983), 717-727.

Mathematical Institute, Slovak Academy of Sciences, Štefánikova 49, 81473 Bratislava, Slovakia

E-mail address: matefeck@savba.savba.sk 